\title{
Research Progress in Biological Package for Aquaculture Wastewater Treatment
}

\author{
Xi-dong MU, Yin-chang Hu (Corresponding author), Guang-jun Wang, Jun Chen \& Jian-ren Luo \\ Key laboratory of Fishery Equipment and Engineering \\ Ministry of Agriculture of the People's Republic of China \\ Shanghai 200092, China \\ $\&$ \\ Pearl River Fishery Research Institute, Chinese Academic of Fishery Science \\ Guangzhou 510380, China \\ Tel: 20-2217-5232Ｅ-mail:muxd1019@126.com
}

This study was supported by Open Fund of Key laboratory of Fishery Equipment and Engineering, Ministry of Agriculture of the People's Republic of China( No.2009010) and Science and Technology Program of liwan district Guangdong Province(No.20100065).

\begin{abstract}
The classification of biological package was reviewed in the present paper, and the application status of various fillers for aquaculture wastewater treatment was introduced in detail. The developing direction of biological package in the field of aquaculture wastewater treatment was also presented.
\end{abstract}

Keywords: Biological package, Classification, Aquaculture wastewater treatment, Developing direction

\section{Introduction}

With the rapid development of the fisheries, aquaculture species have degraded, especially for high-density aquaculture of random discharge of industrial wastewater and domestic sewage so that capacity of self-purification and regulation of ponds reduced, and water environment exacerbated. For instance, baits and feces entered into the water in the farming mode of main feeding, so that the amount of ammonia produced in aquiculture, sub-nitrate nitrogen, chemical oxygen demand and biological oxygen demand severely exceed the standard. Ameliorating the water environment of aquaculture has become the key technology and research focus for sustainable development of breeding production.

Using the attachment growth on solid surfaces of microbial to undertake wastewater biological treatment, has resulted in the emergence of biological package. Its material, specific surface area, water and air distribution performance, strength, density, cost and other factors, had a direct impact on wastewater processing efficiency and operational feasibility. Along with the development of science and technology related to biological package and the further investigation on membrane structure, mechanism, growth dynamics principle and other areas of biological package imposed by domestic and foreign researchers, various types of biological package continuously varied and upgraded, gradually enhanced the attachment capabilities of biological package to biofilm, and thus improved the efficiency of biofilm treatment.

\section{Classification and characteristics of biological package}

Biological package could be approximately classified into several sorts, such as fixed type, suspension type, decentralized type, new biological package and so on (Zhang, 2004, PP. 8-12; Jiang, 1994, PP. 13-16).

Fixed type package was mainly rigid filler, including the nest-shaped, corrugated plate, filling home-style and other fillers. Its characteristics were depicted as follows: porosity was large; wastewater could three-dimensional flow in the filler; filling materials was light and tough without degradation after long-term use; mesh honeycomb filler interaction had a long stability. Its disadvantage was easy to jam.

Suspension type was very commonly used fillers in the overfilling process of biological wastewater treatment, and was generally classified into soft filler, semi-soft filler, combined packing flexible packing and other fillers. Among them, soft filler was applied intensively and extensively. Its hanging film was easy with non-blocking, but was easy to tangle, caking, broken wires, and the central rope was easily broken, which affected its service 
life. Combined packing possessed the chemical properties of resistance to acid, alkali, aging and biological degradation, and physical properties of large surface area, light weight and attachment capabilities of fillers to biofilm. It also exerted the advantages of semi-soft filler such as no caking, strong ability of redistributing air and water and high efficiency of oxygen transfer.

Decentralized type contained accumulated filler and suspended filler. Accumulated filler mainly consisted of accumulation of ceramic, activated carbon and other filter bed packing. Suspended filler has been in development, with considerable sorts, and mainly included no non-woven fabric, polyhedron empty ball, inner-placed suspended ball, SNP suspension (Zhang, 1995, PP. 180-183) and SQC filamentous ball-like suspension fillers and other packing. Its characteristics were that it was not necessary to fix and install with large surface area. Its detachment process was easy, could be continuously rinsed in the constant movement and thus was hard to jam. It showed an obvious effect on the repeated cutting of air bubbles when operated in the water, therefore, dissolved oxygen value in the water increased promptly, oxygen transfer efficiency was great, and microbial metabolism thrived.

New biological package mainly contained immobilized microorganisms and bionic-type fillers. As for immobilized microorganisms, it could be presented that microorganisms were fixed using a certain methods, and were applied as a solid catalyst. Carrier which had good immobilized effects on microorganisms exactly called immobilized microorganisms fillers; Bionic-type fillers were some packing which were processed and made by simulating natural aquatic form. Its density was similar to water, and suspended in the water. Therefore, it was new type suspended filler.

\section{Effects of fillers on biological aerated filter efficiency}

Filler was the key to biological aerated filter. In order to enhance the performance of biological aerated filter, fillers should be selected, and the particle size and height of fillers should be optimized; investigating and developing new type fillers and their corresponding filters was the effective approach to enhance biological aerated filter efficiency.

\subsection{Effects of filler particle size}

Filler particle size was the important parameter affecting the processing performance of filter. Kent et al (Kent, 2000, PP. 409-414) studied the effects of biological aerated filter on the removal of ammonia nitrogen, and indicated that filler particle size of $2 \mathrm{~mm} \sim 4 \mathrm{~mm}$ had a far better effects on nitrification performance of biological aerated filter than of $4 \mathrm{~mm} \sim 8 \mathrm{~mm}$, or $5.6 \mathrm{~mm} \sim 11.2 \mathrm{~mm}$. Filler particle size had a significant impact on the processing performance and operating period of biological aerated filter. The smaller filler particle size was, the better effects the treatment had. But when filler particle size was smaller, filter was easy to jam, its operating period was comparatively short which required the repeated rinsing, and thus it was hard to exert the further effects of fillers. Therefore, when selecting fillers for biological aerated filter, processing performance and operating period of filter should be taken into consideration, and undertaken optimizing selection according to the influent quality of filter and processing requirements.

\subsection{Effects of filler density}

Filler density of biological filter was associated with backwashing intensity. The larger the density was, the stronger backwashing intensity was, and the bigger required energy consumption was; filter density was lower, and consumption energy for backwashing correspondingly decreased. Consequently, in the selection of fillers for biological filters, densities of various fillers should be measured.

\subsection{Effects of packing height}

Packing height of biological aerated filter had a considerable direct effect on removal effects and infrastructure investment. Francisco et al (2001, PP. 974-978) have investigated the relationship between work performance and height of downfall biological aerated filter, and thought that proper height of bed layer using ceramic as filler for biological aerated filter should be ranged from $1.2 \mathrm{~m}$ to $1.5 \mathrm{~m}$. Different dominant flora could be formed along with the flow direction in biological aerated filter. Packing height was related to the effluent quality, and increasing packing height could enhance the processing effect of filters and guarantee the effluent quality within certain extent, but at the same time, it could also increase the lifting and backwashing intensity of wastewater, and thus induce more energy consumption.

\section{Applications of biological packing in the wastewater treatment}

Various fixed type and suspension type fillers were widely applied in the micro-polluted water treatment. Ji Rongping (2005), Mei Xiang et al (1999, PP. 1-4), Fan Cijiao et al (1996, P. 11), Yang Zaigao et al (1997, PP. 
2-5) have studied various fillers respectively, and found that its removal rate of ammonia nitrogen in all kinds of water sources could basically attained more than $40 \%$, and the top value could be up to $95 \%$; its removal rate of CODMn could basically attained more than $10 \%$, and the top value could be up to $54 \%$; it also had a high removal rate of turbidity, color and algae.

In the applications of accumulated fillers, many studies (Huang, 1998, PP. 60-62; Huang, 1996, PP. 15-20; Luo, 1996, PP. 12-15) have indicated that biological contact oxidation pond using bio-ceramic as fillers in the treatment of multiple sources of water had achieved good results. Its removal rate of ammonia nitrogen in all kinds of water sources was quite high; its removal rate of $\mathrm{COD}_{\mathrm{Mn}}$ could basically attain more than $15 \%$, and the top value could be up to $60 \%$; it also had a high removal rate of turbidity and color.

In recent years, activated carbon has a large specific surface area and well developed pore structure. It could not only adsorb micro-pollutants in water, but also be an ideal habitat for aquatic microorganisms. Therefore, it was applied in the water treatment, which could exert its dual effects, viz. physical adsorption and microorganism biodegradation, and formed the process of biological activated carbon (Wang, 2007, PP. 1-4). This process would get more and more applications in wastewater treatment. More recently, researchers have begun to study and prepare hydrophilic modified plastics used for biological fillers, for instance, hydrophilic substances were induced into polypropylene and polyvinyl chloride with attempts to achieve hydrophilic modification. If hydrophilic polymer materials such as polyvinyl alcohol and polyacrylamide were induced into plastics, in order to ameliorate materials' surface hydrophilic properties and create plastic materials with hydrophilic surface, and thus hydrophilic elastic filler was invented for water treatment. Such hydrophilic fillers and general fillers with the same shape and structure and different materials were undertaken hydrophilic test using weighing method, each filler was weighed after soaking for $1 \mathrm{~min}, 30 \mathrm{~min}, 1 \mathrm{~d}$ and $8 \mathrm{~d}$, respectively, and results showed that the patent filler hold greater weight than general filler in the same soaking time; these two kinds of fillers were undertaken anaerobic and aerobic treatment by biofilm method using simulated wastewater, and results showed that hydrophilic filler had a better effect on the removal of CODCr than general filler biofilm reactor; anaerobic biofilm was undertaken shock loading and recovery experiments, and results showed that biofilm in biofilm reactor with hydrophilic filler had a stronger resistance to shock loading, and needed a short recovery time after shocking.

\section{Future developing direction of aerated filter fillers:}

(1) Study the impact of fillers on the removal of pollutants and its removal mechanism. Currently, there have been many investigations on process conditions of biological aerated filter operating, but less further researches on the transfer and metabolic pathway of pollutants; biofacies and ecological structure of fillers lacked systematic analysis and interpretation; In addition, effects of fillers on the removal of pollutants still remained unclear.

(2) Develop inorganic fillers with the main component of natural materials, such as study and development of light weight aggregate. Compatibility between the synthetic polymer filler and microbes were quite poor, so biomass was little and easy to drop during biofilm colonization, while inorganic filler, using natural materials as raw material, could overcome the abovementioned shortcomings, but the key problem which should be solved immediately was to increase the strength, increase porosity and decrease density.

(3) Seek processes and methods to improve the performance of fillers. The processing of fillers was extremely important, and in order to obtain the packing with excellent quality, producing processes and methods processes should be continuously ameliorated.

(4) Establish the filler standard suitable for biological aerated filter. Europe and the United States all had very stringent standards on the fillers used in biological aerated filters, but in China, there are no one until now. After systematic grasping effects of filler factors such as size, character, density and so on, to establish filler standard suitable for biological aerated filters in China was of profound significance. This could not only make filler production standardization, but also greatly promote the application and extension of wastewater treatment technology of biological aerated filters in China.

\section{References}

Fan, C.J., Chen, K.X., \& Shen, J. (1996). Aquatic biological treatment trial in the post channel water source of Zhujiang River. Water \& Wastewater Engineering, 22(11):11-11.

Francisco, O., \& Emesst, H. (2001). Optimization of bed material height in a submersed biological aerated filter. Journal of Environment Engineering, 974-978.

Huang, X.D., Cao, T.H., \& Tan, W.M., et al. (1998). Investigation on bio-ceramic in the water treatment in 
Shenzhen reservoirs. Environmental Science, (6):60-62.

Huang, X.H., Wang, Z.S., \& Song, B.C. (1996). Study on the biological contact oxidation pretreatment of Chaohu Lake raw water. Water \& Wastewater Engineering, 22(8):15-20.

Ji, R.P. (2005). Effects of artificial medium on ameliorating the water quality of Taihu water source and its mechanism. Dissertation Submitted to Southeast University in Partial Fulfillment of the Requirement for the Degree of Doctor.

Jiang, Z.H. (1994). Types, performance and development trend of fillers in wastewater treatment. Environmental Pollution \& Control, 16 (4):13-16.

Kent, T.D., Williams, S.C., \& Fitzpatrick, C.S.B. (2000). Ammonic nitrogen removal in biological aerated filters: the effect of media size. J. CIWEM, 14: 409-414.

Luo, X.H., Wang, Z.S, \& Wang, H., et al. (1996). Biological Pretreatment of eutrophic lake water. Water \& Wastewater Engineering, 22(7):12-15.

Mei, X., Chen, H.B., \& Gao, T.Y., et al. (1999). Preliminary comparison of treatment effects of several sorts of fillers in the micro-polluted source water biological oxidation process. Water \& Wastewater Engineering, 25(5):1-4.

Yang, Z.G. (1997). Phase summary of removing pollutants from eutrophicated raw water in Taihu Lake. Water Purification Technology, 61 (3):2-5.

Zhang, F., Cheng, J., \& Yang, Z.R., et al. (2004). Research progress in biofilm carrier for wastewater treatment. Techniques and Equipment for Environmental Pollution Control, 5(4):8-12.

Zhang, H.M., \& Wen, Y.B. (1995). Design and using method of SNP suspended fillers. Environmental Protection of Chemical Industry, (15):180-183.

Wang, L., Wang, L., \& Li, F.T., et al. (2007). Developments and the application of biological activated carbon to water treatment. Industrial Water Treatment, 27(7):1-4. 